\title{
2-Deoxy-d-glucose: from diagnostics to therapeutics
}

\section{Rajeev Goel*}

Department of Biophysics, Dr. Rajendra Prasad Govt. Medical College, Kangra, Himachal Pradesh, India

Received: 09 May 2021

Accepted: 21 May 2021

\section{*Correspondence:}

Dr. Rajeev Goel,

Email: rgoel302@yahoo.com

Copyright: (c) the author(s), publisher and licensee Medip Academy. This is an open-access article distributed under the terms of the Creative Commons Attribution Non-Commercial License, which permits unrestricted non-commercial use, distribution, and reproduction in any medium, provided the original work is properly cited.

\begin{abstract}
Glucose is the most common source of cellular energy and a substrate for many biochemical processes. Abnormal glucose signalling has been found in many diseases including cancers and inflammatory diseases. A glucose analog, 2deoxy-D-glucose (2DG) which interferes with the cellular glucose metabolism has shown promising results both as a diagnostic and therapeutic agent in certain diseased conditions such as cancer, cardiovascular diseases, Alzheimer disease, etc. Viral-infected cells have also been found to increase their glucose uptake and recently Drug Controller General of India (DCGI) has given approval for the therapeutic use of 2DG in managing severe acute respiratory syndrome corona virus 2 (SARS-CoV-2) infection which has received worldwide attention and has been declared a public health emergency of global concern. An attempt has accordingly been made in this article to review the diagnostic and therapeutic potentials of 2-deoxy-glucose with special emphasis on to its implications in SARS-CoV-2.
\end{abstract}

Keywords: Glycolysis, 2-deoxy-D-glucose, SARS-CoV-2, Anticancer and antiviral therapy

\section{INTRODUCTION}

The Institute of Nuclear Medicine and Allied Sciences (INMAS), New Delhi, a lab of Defence Research and Development Organisation (DRDO), in collaboration with its industry partner Dr Reddy's Laboratories (DRL), Hyderabad, India has very recently announced antiCOVID-19 therapeutic applications of the oral drug 2deoxy-D-glucose (2-DG).

It is claimed that the drug will be of great benefit due to its effectiveness not only in speedy recovery but also reduced dependence on supplemental oxygen as seen in clinical trials on people suffering from COVID-19. The higher percentage of patients treated with 2-DG revealed reverse transcription polymerase chain reaction (RT-PCR) negative conversion in COVID cases. The Drug Controller General of India (DCGI) based on these results consequently granted approval on 01 May 2021 for emergency use of this drug as adjunct therapy in moderate to severe COVID-19 patients as per the press release of Ministry of Defence, Government of India. ${ }^{1}$

\section{WHAT IS 2-DEOXY-D-GLUCOSE?}

Glucose is the most common source of cellular energy and a substrate for many biochemical processes. 2-DG is an analogue of glucose molecule having 2-hydroxyl group replaced by hydrogen (Figure 1) which prevents it to undergo glycolysis. ${ }^{2}$. 2-DG act as a D-glucose mimic. It is an anti-metabolite which inhibits glycolysis due to formation and intracellular accumulation of 2-deoxy-Dglucose-6-phosphate (2-DG6P) thereby inhibiting the function of hexokinase, glucose-6-phosphate isomerase and production of adenosine triphosphate (ATP) (Figure 2). ${ }^{3,4}$ The other molecular processes such as activating adenosine-activated protein kinase (AMPK) causing induction of autophagy; activation of tumor suppressor gene p53 leading to apoptosis; protein N-glycosylation resulting in endoplasmic reticulum (ER) stress are also modulated by 2-DG in addition to inhibition of 
glycolysis. ${ }^{5-11} 2-\mathrm{DG}$ is transportable across the blood brain barrier (BBB) similar to D-glucose and quickly taken up into cells by facilitated diffusion mainly through glucose transporters, in particular glucose transporter 1 (GLUT1) and GLUT4. ${ }^{12}$

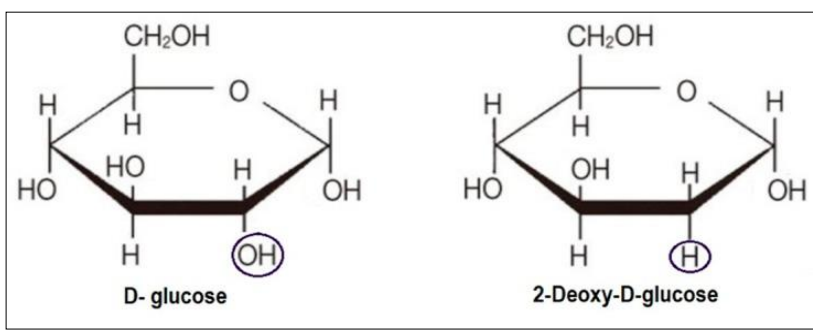

Figure 1: Chemical structure of D-glucose and 2-DG, the $\mathrm{OH}$ group position is encircled in 2 -DG.

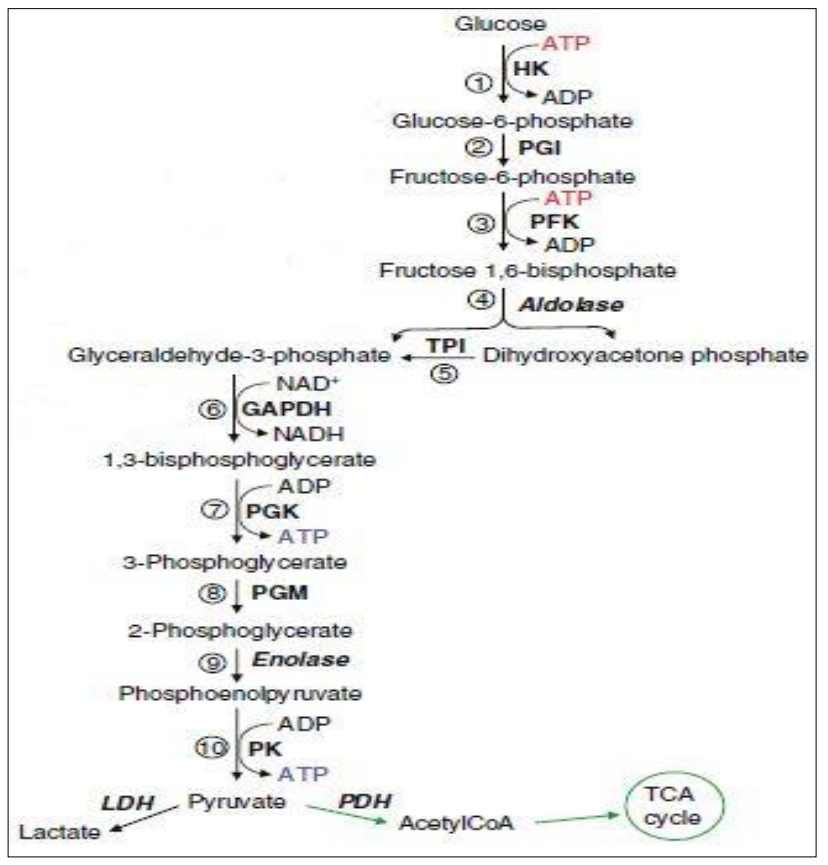

Figure 2: Glycolytic pathway.

HK-hexokinase; PGI-phosphoglucose isomerase; PFKphosphofructokinase; TPI-triosephosphate isomerase; GAPDHglyceraldehyde-3-phosphate dehydrogenase; PGKphosphoglycerate kinase; PGM-phosphoglycerate mutase; PKpyruvate kinase; PDH-pyruvate dehydrogenase; LDH-lactate dehydrogenase

\section{DIAGNOSTICS APPLICATIONS \\ AND \\ THERAPEUTICS}

Attempts have been made to use 2-DG both as the diagnostic and therapeutic agent. The radiolabelled 2-DG when used in positron emission tomography (PET) can determine glucose metabolism which is altered in diseases such as cardiovascular disease, Alzheimer's disease and tumors. ${ }^{13}$ The labelled form (fluorine-18) of 2-DG $\left({ }^{18} \mathrm{~F}\right.$ DG or FDG) serve as a good marker for tissue glucose uptake as many cancers have elevated glucose uptake due to increased cellular proliferation. ${ }^{14} 2-\mathrm{DG}$ is taken up by the glucose transporters of the cell. Therefore, cancerous cells with higher glucose uptake have an increased uptake of 2-DG.

2-DG therapeutically is an investigational drug that is studied as an anticancer and antiviral agent. ${ }^{15}$ 2-DG showed potential indications as an adjunct to chemotherapy and radiotherapy in the treatment of solid tumors such as breast, head, neck, lung, pancreas, gastric and lung tumors. ${ }^{16,17}$ 2-DG has been shown to sensitize cancer cells to cytotoxic drugs to which they are resistant when used alone. ${ }^{18,19}$

Most of the cancers display elevated dependency on aerobic glycolysis, a phenomenon referred as the "warburg effect". ${ }^{20}$ Tumor glucose metabolism in particular is a promising target, since it strongly differs from glucose metabolism in normal cells. ${ }^{21}$ The non-metabolizable analogues of glucose, like 2-DG has been put forward to be of immense therapeutic use in cancer treatment. It may be because of the fact that 2-DG competitively inhibits glucose transport inhibiting glycolysis leading to energy depletion, hampers glycosylation of certain putative regulatory proteins, cell cycle arrest and, ultimately cell survival. ${ }^{10,17,22}$ One of the major hurdles, however, in using 2-DG as a chemotherapeutic agent is that it can induce endoplasmic reticulum stress (ER stress). ER stress is a distinctive feature in many solid tumors and the unfolded protein response (UPR) associated with it initiates many survival mechanisms in cancer cells..$^{9,10,23}$

A clinical trial demonstrated that 2-DG can be tolerated at a dose of $63 \mathrm{mg} / \mathrm{kg} /$ day. The phase I trial to evaluate the safety, pharmacokinetics (PK) and maximum tolerated dose (MTD) recommended that $63 \mathrm{mg} / \mathrm{kg} /$ day dose of 2DG in combination with weekly docetaxel is clinically tolerable dose in patients with advanced solid tumors with no serious side effects or with tolerable adverse effects such as fatigue, sweating, dizziness and nausea mimicking the hypoglycemic symptoms expected from 2-DG administration. ${ }^{24}$ There was no pharmacokinetics interaction between 2-DG and docetaxel. It however at higher doses $(63-88 \mathrm{mg} \%)$ leads to gastrointestinal bleeding $(6 \%)$, cardiac side-effects such as prolongation of the Q-T interval (22\%) and cancer progression in majority of the cancer patients $(66 \%){ }^{24}$ This raised doubts on the viability of this reagent for clinical use. However, it was also observed in one of the studies carried on brain tumor patients that most of the side effects of oral administration of 2-DG (up to doses of $250 \mathrm{mg} / \mathrm{kg} \mathrm{b.w}$.) are transient and reversible. ${ }^{25}$ More studies are nonetheless underway to fully explore the use of 2-DG as chemotherapeutic agent.

2-DG shows great promise as a new anti-epileptic drug, as treatment for epilepsy have investigated the role of glycolysis in the disease. ${ }^{26} 2-\mathrm{DG}$ in temporal lobe epilepsy represses the overactive brain-derived neurotrophic factor (BDNF) gene promoter. It consequently prevents the increased expression of BDNF protein and TrkB receptor (BDNF's receptor) that is observed in epileptic patients. ${ }^{26}$ 
The study on herpes simplex virus-1 (HSV-1), though a relatively large and complex double stranded DNA virus unlike corona virus, has demonstrated that the production of infectious herpes simplex 1 was reduced $94-98 \%$ by growth when exposed to $6 \mathrm{mM} 2-\mathrm{DG}$ in vitro in vero cell lines. The HSV-1 when grown in the presence of 2-DG was capable of binding to vero cells almost parallel to control virus. However, the rate as well as amount of penetration was markedly reduced for virus grown with 2DG than for control virus. ${ }^{27}$ The study concluded that modification in the capacity to penetrate the cell surface of virus grown in the presence of 2-DG is a significant factor in the antiviral action of 2-DG.

The study by Passalacqua et al also observed the antiviral effects of 2-DG. The anti-viral effect was accredited to the direct interaction of 2-DG with the murine noroviruses (known to causes gastroenteritis) which inhibited viral entry into host macrophages cells and weakened the high energy demand by inhibition of glycolysis. The study suggested glycolysis as a virulence determinant. ${ }^{28}$

2-DG is known to exert anti-inflammatory effects. ${ }^{29} 2-\mathrm{DG}$ suppressed immune cell activation and reduced disease severity in an autoimmune model of rheumatoid arthritis. ${ }^{30}$ Although there exists several possible therapeutic indications for 2-DG, there is no approved indication for 2-DG as a therapeutic agent so far. It seems that most promising application of 2-DG may be as a synergistic agent in combination with cytotoxic therapies. ${ }^{31,32}$

\section{2-DG AND SARS-CoV-2}

Host cells are highly dynamic systems that can alter their intracellular environment and metabolic behavior, which may be helpful or inhibitory for an infecting virus. Viruses take control of the host cell metabolism so as to have optimal environment for their rapid and efficient replication and transmission. One salient example is an augmented uptake of vital nutrients such as glucose to support metabolic signalling, i.e. aerobic glycolysis which is a principal pathway of glucose metabolism and its byproducts for biosynthetic reactions. ${ }^{33}$

The inhibition of influenza B virus multiplication, in intact chick embryo inoculated by the allantoic route, with glucose anti metabolite 2-DG was shown long back in 1959 by Kilbourne from Cornell University Medical College, New York. ${ }^{34}$ In parallel it was also demonstrated by certain ancillary studies with in vitro systems that the inhibition of influenza multiplication to be reversible with glucose and therefore not related to the damage to the host cells. 2-DG also results in a disruption of rhinovirus (RV) replication in vitro (HeLa cell lines, human fibroblasts) and in vivo (femaleC57BL/6 J mice). RV-infected cells rapidly up-regulate glucose uptake by increasing the expression of the PI3K-regulated glucose transporter GLUT1. The glycolysis inhibition by 2-DG potently impairs rhinovirus replication. ${ }^{34}$
The recent studies by different researchers have shown that inhibition of glycolysis by 2-DG and inhibition of glycolytic enzymes 6-phospho-fructo-2-kinase/fructose-2, 6-bisphosphatase-3 (PFKFB3), a positive regulator of phosphofructokinase-1 (PFK1) and lactate dehydrogenase A (LDH-A) terminates viral replication and cytokine response. ${ }^{35}$ This puts glycolysis as a requisite upstream event during SARS-CoV-2 pathogenesis though crucial glycolytic intermediate/final product (s) regulating it are not very well defined. It has been analogously observed that 2-DG blocks SARS-CoV-2 replication in a colon epithelial carcinoma cell line as well. ${ }^{36}$

It is pertinent to note that metabolic transcription factor hypoxia-inducible factor- $1 \alpha$ (HIF-1 $\alpha)$, is a master regulator of glycolysis which along with its target genes are greatly induced in monocytes infected with SARSCoV-2. ${ }^{35}$ The HIF- $1 \alpha$ inhibition or stabilization is known to either block or heighten HIF-1 $\alpha$ target genes, viral replication, angiotensin converting ezyme-2 (ACE-2) and cytokines expression in SARS-CoV-2 infected human monocytes under diabetic condition indicating that HIF- $1 \alpha$ is vital for elevated glycolysis and thereafter inflammatory responses. ${ }^{35}$

The studies to explore the mechanistic insight into the stabilization of HIF-1 $\alpha$ under increased glucose condition concluded that an increase in mitochondrial reactive oxygen species (ROS) due to impaired oxidative metabolism is the cause for HIF-1 $\alpha$ stabilization and the pro inflammatory state in SARS-CoV-2 infected monocytes. ${ }^{35,37}$

The study consequently proposed that SARS-CoV-2 infection boost glycolysis by abnormal production of mitochondrial ROS in monocytes cultured under high glucose concentration. It subsequently results in activation of transcription factor hypoxia-inducible factor- $1 \alpha$ (HIF$1 \alpha$ ), which warrants the fast replication of the virus. The increased glucose metabolism through aerobic glycolysis, therefore, nurtures viral replication and cytokine production weakening $\mathrm{T}$-cell response and function and induces lung epithelial cell death which may be the cause of diabetic people to be more susceptible to severe COVID 19 infection. $^{35-37}$

People with COVID-19 have raised pro-inflammatory cytokine IL-1 $\beta$ which in turn is modulated through generation of 3-phosphoglycerate, a by-product of glycolysis. This suggests a direct link between IL-1beta and glucose metabolism which is substantiated by the recent study conducted in mice wherein the inhibition of glycolysis with 2-DG is shown to stop IL-1 $\beta$ induction and to protect against LPS-induced sepsis. ${ }^{37,38}$

The computer simulation and docking studies by the Patanjali group demonstrated that 2-DG structurally docks well into viral protease 3CLpro and NSP15 endoribonuclease causing the inhibition of SARS-CoV-2 receptors binding to the host cells. ${ }^{39}$ This, however, needs 
experimental confirmation (Thakur et al). The virus main protease 3CLpro and NSP15 endonuclese are known to take control of the host machinery. The in silico study supported the possible therapeutic benefits of using 2-DG as repurposed drug/pro drug for diminishing the novel COVID-19 infection (Figure 3). This is the first published study that directly evaluates in silico the COVID-19 sites that $2-\mathrm{DG}$ could have important anti-viral activity on. ${ }^{39}$

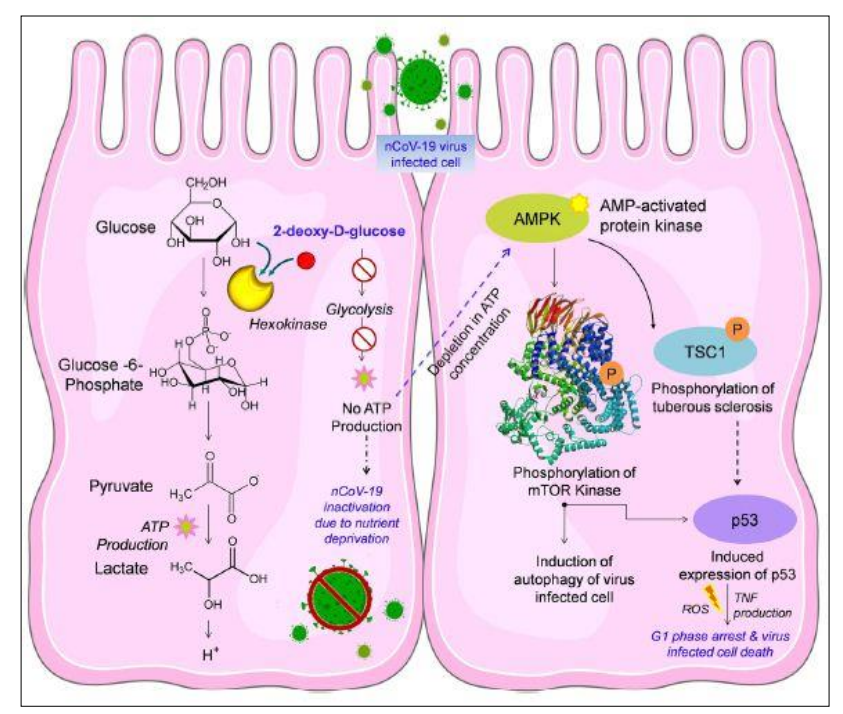

Figure 3: Diagramatic representation of effect of 2DG on glycolysis inhibition, ATP depletion, ROS production, cell cycle arrest ultimately leading to inactivation of the $\mathrm{nCoV}-19$ virus and the virus infected cell death. ${ }^{39}$

The plethora of pharmacological effects of 2-DG such as anti-inflammatory action, inhibition of cellular glycolysis and interaction with viral proteins triggered the possibility of 2-DG to be used as a therapeutic agent for the management of COVID-19 patients. ${ }^{40} 2-\mathrm{DG}$ lowered the viral load by 1-2 logs in avian corona virus infected cells in vitro at 3 and $10 \mathrm{mM}$ concentration in preliminary experiment conducted in March, 2020 at Pirbright Institute in the UK. ${ }^{40}$ The studies exist wherein the potentiality of $2-\mathrm{DG}$ to enhance the antigen restoring $\mathrm{CD} / \mathrm{CD} 8$ ratio, boost NK cells and IFN presenting ability of macrophages were observed which implicated that 2-DG may also revamp the immune status compromised by COVID-19. ${ }^{41-}$ 43 The various studies however raised doubts about the dose of 2-DG required and daily administration needed which may precipitate the non-target effects in the form of CNS and cardio-respiratory disturbances. ${ }^{44-46}$

\section{CONCLUSION}

We have in recent years seen remarkable progress in research on 2-DG to be used both for diagnostic and therapeutic purposes. The latest studies on 2-DG inhibitory effect on SARS-CoV-2 together with the anti-viral activity of 2-DG previously reported in a number of different virus types as discussed in this article add support to therapeutic applications of the drug 2-DG against SARS-CoV-2 as claimed by INMAS, New Delhi. It is believed that enough care has been taken to test the drug dosage, efficacy and safety profile of 2-DG during the different phases of the clinical trials to help manage the COVID-19 disease keeping in view the ongoing pandemic and its approval for final use on COVID-19 patients as an adjunct therapy.

Funding: No funding sources

Conflict of interest: None declared

Ethical approval: Not required

\section{REFERENCES}

1. DCGI approves anti-COVID drug developed by DRDO for emergency use. Available at https://pib.gov.in/PressReleasePage.aspx?PRID=171 7007. Accessed on 08 May 2021.

2. Wick AN, Drury DR, Nakada HI, Wolfe JB. Localization of the primary metabolic block produced by 2-deoxyglucose. J Biol Chem. 1957;224(2):963-9.

3. Chen $\mathrm{W}$, Guéron $\mathrm{M}$ The inhibition of bovine heart hexokinase by 2-deoxy-D-glucose-6-phosphate: characterization by 31P NMR and metabolic implications. Biochimie. 1992;74:867-73.

4. Demetrakopoulos GE, Linn B, Amos H. Starvation , deoxy sugars, ouabin and ATP metabolism in normal and malignant cells. Cancer Biochem Biophys. 1982;6:65-74.

5. Gareth SH, Brown GJ, K rauseU, Patel JV, Vertommen D, Bertrand L, et al. Activation of AMPActivated Protein Kinase Leads to the Phosphorylation of Elongation Factor 2 and an Inhibition of Protein Synthesis. Curr Biol. 2002;12(16):1419-23.

6. Hardie D, Lin SC. AMP-activated protein kinaseNot just an energy sensor. F1000 Res. 2017;6:1724.

7. Jung CH, Ro SH, Cao J, Otto NM, Kim DH. mTOR regulation of autophagy. FEBS Lett. 2010;584:128795.

8. Shutt DC, O’Dorisio MS, Aykin-Burns N, Spitz DR. 2-deoxy-D-glucose induces oxidative stress and cell killing in human neuroblastoma cells. Cancer Biol Ther. 2010;9:853-61.

9. Kurtoglu M, Gao N, Shang J, Maher J.C, Lehrman M.A, Wangpaichitr M, et al. Under normoxia, 2deoxy-D-glucose elicits cell death in select tumor types not by inhibition of glycolysis but by interfering with N-linked glycosylation. Mol Cancer Ther. 2007;6:3049-58.

10. Xi H, Kurtoglu M, Liu H, Wangpaichitr M, You M, Liu X, Savaraj N, Lampidis, T J. 2-Deoxy-d-glucose activates autophagy via endoplasmic reticulum stress rather than ATP depletion". Canc Chemother Pharmacol. 2010;67(4):899-910.

11. Xi H, Kurtoglu M, Lamipidis T.J. The wonders of 2deoxy-D-glucose. IUBMB J. 2014;66:110-21.

12. Navale AM, Paranjape AN. Glucose transporters: physiological and pathological roles. Biophys Rev. 2016;8(1):5-9. 
13. Mees G, Dierckx R, Vangestel C, Laukens D, Damme NV, De Wiele CV. Pharmacologic Activation of Tumor Hypoxia: A Means to Increase Tumor 2Deoxy-2-[F-18] Fluoro-D-Glucose Uptake? Mol Imaging. 2013;12:49-58.

14. Viswanathan C, Faria S, Devine C, Patnana M, Sagebiel T, Iyer RB, Bhosale PR. [F]-2-Fluoro-2Deoxy-D-glucose-PET Assessment of Cervical Cancer. PET Clin. 2018;13:165-77.

15. Kang HT, Hwang ES. 2-Deoxyglucose: An anticancer and antiviral therapeutic, but not any more a low glucose mimetic. Life Sci. 2006;78(12):1392-9.

16. Gupta S, Farooque A, Adhikari JS, Singh S, Dwarakanath BS. Enhancement of radiation and chemotherapeutic drug responses by 2-deoxy-Dglucose in animal tumors J Can Res Ther. 2009;5:1620.

17. Aft RL, Zhang FW, Gius D. Evaluation of 2-deoxy-Dglucose as a chemotherapeutic agent: Mechanism of cell death. Br J Cancer. 2002;87:805-12.

18. Cao X, Fang L, Gibbs S, Huang Y, Dai Z, Wen P, Zheng $\mathrm{X}$, et al. Glucose uptake inhibitor sensitizes cancer cells to daunorubicin and overcomes drug resistance in hypoxia. Cancer Chemother Pharmacol. 2007;59:495-505.

19. Catanzaro D, Gaude E, Orso G, Giordano C, Guzzo G, Rasola A, Ragazzi E, et al. Inhibition of glucose-6phosphate dehydrogenase sensitizes cisplatin-resistant cells to death. Oncotarget. 2015;6(30): 30102-14.

20. Liberti MV, Locasale JW. The Warburg Effect: How Does it Benefit Cancer Cells? Trends in Biochemical Sciences. 2016; 41(3):211-8.

21. Batra S, Adekola KUA, Rosen ST, Shanmugam M. Cancer metabolism as a therapeutic target. Oncology. 2013;27:460-7.

22. Magier Z, Jarzyna R. The role of glucose transporters in human metabolic regulation. Postepy Biochem. 2013;59:70-82.

23. Defenouillère Q, Verraes A, Laussel C, Friedrich A, Schacherer J, Léon S. The induction of HAD-like phosphatases by multiple signaling pathways confers resistance to the metabolic inhibitor2deoxyglucose.Sci Signal. 2019;12:597.

24. Raez LE, Papadopoulos K, Ricart AD, Chiorean EG, DiPaola RS, Stein MN. A phase I dose-escalation trial of 2-deoxy-d-glucose alone or combined with docetaxel in patients with advanced solid tumors. Cancer Chemother Pharmacol. 2013;71:523-30.

25. Singh, D, Banerji AK, Dwarakanath B S, Tripathi RP, Gupta JP, Mathew TL, et al. . Optimizing cancer radiotherapy with 2-deoxy-D-glucose. Strahlentherapie und Onkologie. 2005;181(8):507-14.

26. Garriga-Canut M, Schoenike B, Qazi R, Bergendahl K, Daley TJ, Pfender R M, et al. 2-Deoxy-D-glucose reduces epilepsy progression by NRSF-CtBPdependent metabolic regulation of chromatin structure". Nat Neurosci. 2006;9(11):1382-7.

27. Spivack JG, Prusoff WH, Tritton TR. A study of the antiviral mechanism of action of 2-Deoxy-D-glucose: normally glycosylated proteins are not strictly required for herpes simplex virus attachment but increase viral penetration and infectivity. Virology. 1982;123:123-38

28. Passalacqua KD, Lu J, Goodfellow I, Kolawole AO, Arche JR, Maddox RJ, et al. Glycolysis is an intrinsic factor for optimal replication of a norovirus. Host Microbe Biology. 2019;10:e02175-18.

29. Choi SY ,Heo MJ, Lee C, Choi YM, An I, Bae S, et al. 2-deoxy-d-glucose Ameliorates Animal Models of Dermatitis. Biomedicines. 2020;8(20)1-11.

30. Abbound G, Choi SC, Kanda N, Spatraro LZ, Roopenian DC, Morel L. Inhibition of glycolysis reduces disease severity in an autoimmune model of rheumatoid arthritis. Front Immunol. 2018;9:1973.

31. Zang F, Aft RL, Chemo sensitizing and cytotoxic effects of 2-deoxy-D-glucose on breast cancer cells. J Cancer Res Ther. 2009;1:41-3.

32. Pajak B, Siwiak E, Sołtyka M, Priebe A, Zieliński R, Fokt I, Ziemniak M, et al. 2-Deoxy-D-Glucose and Its Analogs: From Diagnostic to Therapeutic Agents. Int J Mol Sci. 2020;21(1):234.

33. Sanchez EL, Lagunoff M. Viral activation of cellular metabolism. Virology. 2015;479-80.

34. Kilbourne EB. Inhibition of influenza virus multiplication with a glucose antimetabolite (2-deoxyD-Glucose). Nature. 1959;183:271-2.

35. Codo AC, Davanzo GG , Monteiro LDB, Gabriela Souza GFD, Muraro SP, Virgilio-da-Silva JV, et al. Elevated glucose levels favor SARS-CoV-2 infection and monocyte response through a HIF1alpha/glycolysis-dependent axis. Cell Metab. 2020;32:437-46.

36. Bojkova D, Klann K, Koch B, Widera M, Krause D, Ciesek S, et al. Proteomics of SARS-CoV-2-infected host cells reveals therapy targets. Nature. 2020;583:469-72.

37. Ardestani A, Azizi Z. Targeting glucose metabolism for treatment of COVID-19. Signal Transduction and Targeted Therapy. 2021;6:112.

38. Rodriguez AE, Ducker GS, Billingham LK, Weinberg SE, Rabinowiitz JD, C Chandel NS.Serine metabolism support macrophage il-1B production. Cell Metab. 2019;29(4):1003-11.

39. Balkrishna A, Singh S, DevS, Jain V, Varshney A. Glucose anti metabolite 2-Deoxy-D-Glucose and its derivative aspromising candidates for tackling COVID-19: Insights derived from in silico docking and molecular simulations. 2020.

40. Data Supporting $2 \mathrm{DG}$ as a possible treatment for Covid 19.Lampidis News. Lampidis Cancer Foundation, U.S.A. Available at: www.lampidisfoundation.org. Accessed on 26 March 2020.

41. Farooque A, Afrin F, Adhikari JS, Dwarakanath BS. Polarization of macrophages towards M1 phenotype by a combination of 2-deoxy-d-glucose and radiation: implications for tumor therapy. Immunobiology. 2016;221(2):269-81.

42. Farooque A, Singh N, Adhikari JS, Afrin F, Dwarakanath BS. Enhanced antitumor immunity 
contributes to the radio-sensitization of ehrlich ascites tumor by the glycolytic inhibitor 2-deoxy-D-glucose in mice. PLoS One. 2014;9(9):e108131.

43. Chen G, Wu D, Guo W, Cao Y, Huang D, Wang H, et al. Clinical and immunological features of severe and moderate coronavirus disease 2019. J Clin Invest. 2020;130(5):2620-9.

44. Landau BR, Lubs HA. Animal responses to 2-deoxyD-glucose administration. Proc Soc Exp Biol Med. 1958;99(1):124-7.

45. Vijayaraghavan R, Kumar D, Dube SN, Singh R, Pandey KS, Bag BC, et al. Acute toxicity and cardiorespiratory effects of 2-deoxy-D-glucose: a promising radio sensitiser. Biomed Environ Sci. 2006;19:96-103.
46. Verma A, Adhikary A, Woloschak G, Dwarakanath BS, Papineni RVL. A combinatorial approach of a poly pharmacological adjuvant 2-deoxy-D-glucose with low dose radiation therapy to quell the cytokine storm in COVID-19management. Int $\mathrm{J}$ Rad Biol. 2020;96:11.

Cite this article as: Goel R. 2-Deoxy-d-glucose: from diagnostics to therapeutics. Int J Basic Clin Pharmacol 2021;10:732-7. 